\title{
Spectroscopy of the type la supernova SN 2002er: Days -11 to +215
}

\author{
R. Kotak ${ }^{1}$, W. P. S. Meikle ${ }^{1}$, G. Pignata ${ }^{2}$, M. Stehle ${ }^{3}$, S. J. Smartt ${ }^{4}$, S. Benetti ${ }^{5}$, \\ W. Hillebrandt ${ }^{3}$, D. J. Lennon ${ }^{7}$, P. A. Mazzali ${ }^{3,6}$, F. Patat ${ }^{2}$, and M. Turatto ${ }^{5}$ \\ 1 Astrophysics Group, Imperial College London, Blackett Laboratory, Prince Consort Road, London, SW7 2AZ, UK \\ 2 European Southern Observatory, Karl-Schwarzschild-Str. 2, 85748 Garching bei München, Germany \\ 3 Max-Planck-Institut für Astrophysik, PO Box 1317, 85741 Garching, Germany \\ ${ }^{4}$ Department of Physics and Astronomy, Queen's University Belfast, Belfast, BT7 1NN, Northern Ireland, UK \\ 5 Osservatorio Astronomico di Padova, vicolo dell’Osservatorio 5, 35122 Padova, Italy \\ ${ }^{6}$ Osservatorio Astronomico di Trieste, Via Tiepolo, 11, 34131 Trieste, Italy \\ 7 Isaac Newton Group of Telescopes, Apartado de Correos 321, 38700 Santa Cruz de la Palma, Canary Islands, Spain
}

Received 25 January 2005 / Accepted 14 March 2005

\begin{abstract}
We present an extensive set of optical spectroscopy of the nearby type Ia supernova, SN 2002er, with 24 epochs spanning -11 to +34 days. Its spectral evolution is fairly typical of a type Ia supernova although it suffers high extinction. Nevertheless, there are differences in the spectral evolution when compared to coeval spectra of other normal type Ia supernova with comparable decline-rate parameters. Modelling of the photospheric phase spectra using a homogeneous abundance distribution in the atmosphere provides a fair match to the observations, but only by pushing the adopted distance and risetime close to the observational limits. Future improvements here will require models with a more realistic stratified abundance distribution. From simple modelling of a nebular spectrum obtained at $+215 \mathrm{~d}$, we infer a ${ }^{56} \mathrm{Ni}$ mass of $0.69 M_{\odot}$, consistent with that derived from the light curve.
\end{abstract}

Key words. stars: supernovae: general - stars: supernovae: individual: SN 2002er - methods: observational techniques: spectroscopic

\section{Introduction}

Type Ia supernovae (SNe Ia) are currently the most accurate high- $z$ cosmological distance indicator. Through the light curve shape corrections, accuracies of better than $10 \%$ in distance are claimed (e.g. Phillips et al. 1999; Riess et al. 1998). However, a basic assumption is that the correlations hold at all universal epochs. Yet, even for the best nearby SN Ia sample it appears that the various incarnations of the light curve correction methods may not be wholly consistent with each other (Drell et al. 2000; Leibundgut 2000).

Although there is a general consensus that type Ia supernovae result from the thermonuclear fusion of $\mathrm{C}-\mathrm{O}$ white dwarfs, observational support for the numerous progenitor channels that have been proposed (e.g. Branch et al. 1995) is only just beginning to be gathered (e.g. Ruiz-Lapuente et al. 2004; Hamuy et al. 2003; Kotak et al. 2004). In contrast, there is an increasing body of evidence for the observational diversity (e.g. Benetti et al. 2004b) among type Ia supernovae perhaps suggestive of diversity in the progenitor channels and/or explosion mechanism.
In the favoured scenario leading to a type Ia explosion, the white dwarf is one component of a close binary, and accretes hydrogen from its companion star. Other scenarios include the merging of binary white dwarfs, or the accretion of $\mathrm{He}$ rather than H (e.g. Hillebrandt \& Niemeyer 2000). When the white dwarf reaches the Chandrasekhar limit, explosive carbon burning sets in. Burning to nuclear statistical equilibrium ensues, yielding mostly radioactive ${ }^{56} \mathrm{Ni}$. At lower densities, intermediate-mass nuclei, e.g. ${ }^{28} \mathrm{Si}$, are produced. Thus the typical observed early-time spectra of SNe Ia are dominated by lines of both iron-group and intermediate mass elements. However, the exact nature of the explosion mechanism is uncertain. Competing models can be tested using detailed spectral observations since they are highly sensitive to the nature of the explosion. For example, much higher velocities in intermediate-mass elements are observed in delayeddetonation model spectra than in those of deflagration models (e.g. Khokhlov 1991). Although the optical region is spectroscopically crowded and line-identification is difficult due to the additional complication of blending as a result of Doppler broadening (several thousand $\mathrm{km} \mathrm{s}^{-1}$ ), it is also a region rich in diagnostic potential. For instance, based on 


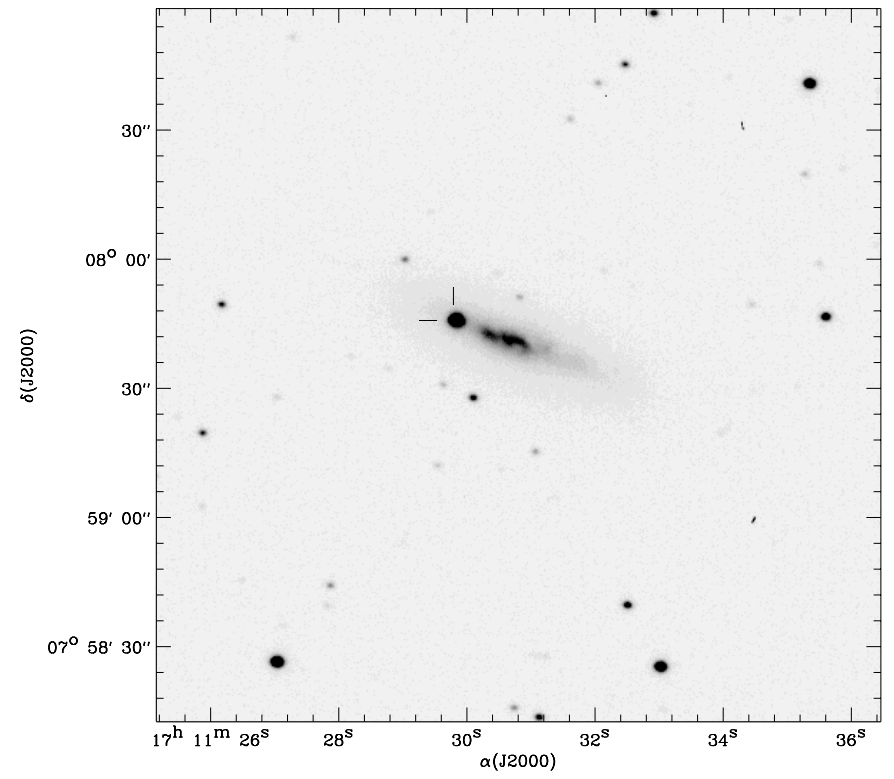

Fig. 1. $B$ band image of SN 2002er taken around maximum light. The dashes indicate the position of the supernova.

measurements and modelling of lines such as SiII $\lambda 6355 \AA$, CII $\lambda 6578, \lambda 7231 \AA$, and the CaII $\mathrm{H}$ and $\mathrm{K}$ lines, Benetti et al. (2004a) argue that the combination of high-velocity intermediate mass elements, an unusually low temperature, and low abundance of carbon indicate that burning to Si penetrated to much higher layers than expected for "normal" type Ia supernovae. This was interpreted as evidence for a delayeddetonation explosion. An alternative scenario could be additional nuclear burning in the so-called distibuted regime at low densities which would have a similar effect (Röpke \& Hillebrandt 2005).

To date, good photometric and spectroscopic coverage extending from pre-maximum light to late times has been obtained for only a handful of supernovae. In order to better understand and quantify the various peculiarities that have been observed, high quality photometric and spectroscopic data from pre-maximum through to nebular epochs is a prerequisite, with detailed comparisons with the predictions of explosion models being made at every step. To this end, the European Supernova Collaboration (ESC) ${ }^{1}$ aims to obtain unprecedented photometric and spectroscopic coverage for $\sim 10$ nearby $\left(v_{\text {rec }} \lesssim\right.$ $4000 \mathrm{~km} \mathrm{~s}^{-1}$ ) type Ia supernovae. The potential of high quality early-time spectroscopy is illustrated by the method of "abundance tomography" (Stehle et al. 2004) whereby the detailed abundance stratification is derived by computing synthetic spectra for over ten epochs, using spectroscopy from the earliest epochs to constrain later ones.

We have already obtained excellent data sets for about eight nearby type Ia SNe. This is the third in a series of observational papers published on behalf of the ESC. We present earlytime optical spectroscopy spanning 24 epochs of the second ESC target, SN 2002er. In a companion paper, Pignata et al. (2004) discuss the photometric evolution of SN 2002er.

\footnotetext{
${ }^{1}$ http://www .mpa-garching.mpg.de/ rtn/
}

Table 1. Properties of SN 2002er and its host galaxy.

\begin{tabular}{ll}
\hline \hline Host galaxy & $\mathrm{UGC} 10743$ \\
Host galaxy type & $\mathrm{Sa}^{\dagger}$ \\
$\mathrm{RA}(2000)$ & $17^{\mathrm{h}} 11^{\mathrm{m}} 29 \mathrm{~s} .88$ \\
Dec $(2000)$ & $+07^{\circ} 59^{\prime} 44^{\prime \prime} 8$ \\
Recession velocity $\left(\mathrm{km} \mathrm{s}^{-1}\right)$ & 2569 \\
Distance modulus* & $32.9 \pm 0.2$ \\
$\left(H_{0}=71 \mathrm{~km} \mathrm{~s}^{-1} \mathrm{Mpc}^{-1}\right)$ & \\
$E(B-V)^{*}$ & 0.36 \\
Offset from nucleus & $12^{\prime \prime} 3 \mathrm{~W} \quad 4^{\prime \prime} \cdot 7 \mathrm{~N}$ \\
Date of $B$ maximum $(\mathrm{MJD})^{*}$ & $52524.2(\mathrm{Sep} .07,2002)$ \\
Magnitude at maximum* & $U=14.72, B=14.89, V=14.59$, \\
& $R=14.43, I=14.49$ \\
$\Delta m_{15}(B)^{*}$ & $1.33 \pm 0.04$ \\
\hline
\end{tabular}

Notes. ${ }^{*}$ Taken from Pignata et al. (2004); $\uparrow$ NED.

\subsection{SN 2002er}

SN 2002er in UGC 10743 was discovered on 2002 Aug. 23.2 UT in NEAT images by Wood-Vasey et al. (2002). and was spectroscopically confirmed to be a type Ia supernova three days later by Smartt et al. (2004); comparison with SN 1994D suggested that it was discovered at an early phase (about $-10 \mathrm{~d}$ before maximum light) and that it was significantly redder. A list of basic parameters is given in Table 1 .

\section{Data acquisition and reduction}

The photospheric-phase observations span -11 to +34 days. Somewhat unusually, the spectroscopic monitoring began several days before the photometric campaign. Optical spectra of SN 2002er were obtained from several different telescopes (Table 2). The observations were carried out in service or target-of-opportunity mode usually using the configuration available at the time. This meant that the set-up varied with epoch and even with observations performed using the same instrument (see Table 2).

The reduction of the data was carried out using standard NOAO IRAF and FIGARO routines (Shortridge 2002). The CCD frames were debiased and corrected for distortion and cosmic rays. Where a sufficient number of flatfield frames were available, the data were also flatfielded. Sky subtraction was carried out by fitting low-order polynomials to the background on either side of the supernova spectrum and optimally extracting one-dimensional spectra from the two-dimensional frames. The wavelength solution was derived using arc frames and was checked against the bright night-sky emission lines. Flux calibration was carried out with respect to spectrophotmetric standards usually taken using an identical instrument configuration and reduced as described above. No attempt was made to correct for the telluric absorption features between $6000-8000 \AA$.

To obtain full wavelength coverage in the optical region, an observation at any given epoch typically consisted of two or three spectra taken either with a single grating/grism at different central wavelengths or using different gratings/grisms. Severe distortion between the blue and red parts of the optical 
Table 2. Log of spectroscopic observations of SN 2002er.

\begin{tabular}{|c|c|c|c|c|c|}
\hline Date & MJD & $\begin{array}{l}\text { Epoch* }^{*} \\
\text { (d) }\end{array}$ & $\begin{array}{l}\text { Approx. range } \\
(\AA)\end{array}$ & $\begin{array}{l}\text { Telescope+Instrument+ } \\
\text { Grating/Grism }\end{array}$ & $\begin{array}{l}\text { Flux } \\
\text { Standard }\end{array}$ \\
\hline 20020826 & 52512.93 & -11.3 & $3500-7200$ & $\mathrm{INT}+\mathrm{IDS}+\mathrm{R} 400 \mathrm{~V}$ & HD 19445 \\
\hline 20020828 & 52514.87 & -9.3 & $3600-7800$ & INT+IDS+R632V & Kopff 27 \\
\hline 20020829 & 52515.86 & -8.3 & $3300-8900$ & INT+IDS+R632V & $\cdots$ \\
\hline 20020830 & 52516.84 & -7.4 & $3600-9000$ & $\mathrm{~A} 1.82+\mathrm{AFOSC}+\mathrm{gm} 2,4$ & $\mathrm{BD}+284211$ \\
\hline 20020831 & 52517.89 & -6.3 & $3400-9900$ & INT+IDS+R632V & Kopff 27 \\
\hline 20020901 & 52518.85 & -5.3 & $3200-9100$ & $\mathrm{CA}+\mathrm{CAFOS}+\mathrm{B}, \mathrm{R} 200^{\dagger}$ & $\mathrm{BD}+284211$ \\
\hline 20020902 & 52519.88 & -4.3 & $3250-9300$ & $\mathrm{INT}+\mathrm{IDS}+\mathrm{R}^{2} 32 \mathrm{~V}^{\dagger}$ & Kopff 27 \\
\hline 20020903 & 52520.85 & -3.3 & $3200-10000$ & $\mathrm{CA}+\mathrm{CAFOS}+\mathrm{B}, \mathrm{R} 200^{\dagger}$ & $\mathrm{BD}+284211$ \\
\hline 20020904 & 52521.83 & -2.4 & $3200-10000$ & $\mathrm{CA}+\mathrm{CAFOS}+\mathrm{B}, \mathrm{R} 200^{\dagger}$ & $\mathrm{BD}+284211$ \\
\hline 20020905 & 52522.82 & -1.4 & $3200-10000$ & $\mathrm{CA}+\mathrm{CAFOS}+\mathrm{B}, \mathrm{R} 200^{\dagger}$ & $\mathrm{BD}+284211$ \\
\hline 20020906 & 52523.85 & -0.3 & $3500-10000$ & $\mathrm{CA}+\mathrm{CAFOS}+\mathrm{B}, \mathrm{R} 200$ & $\mathrm{BD}+284211$ \\
\hline 20020907 & 52524.89 & +0.7 & $6200-10000$ & $\mathrm{CA}+\mathrm{CAFOS}+\mathrm{B}, \mathrm{R} 200^{\ddagger}$ & $\mathrm{BD}+284211$ \\
\hline 20020908 & 52525.88 & +1.7 & $3700-9300$ & $\mathrm{CA}+\mathrm{CAFOS}+\mathrm{B}, \mathrm{R} 200^{\dagger}$ & $\mathrm{BD}+284211$ \\
\hline 20020910 & 52527.81 & +3.6 & $3200-10000$ & $\mathrm{CA}+\mathrm{CAFOS}+\mathrm{B}, \mathrm{R} 200^{\dagger}$ & $\mathrm{BD}+284211$ \\
\hline 20020911 & 52528.81 & +4.6 & $3450-7600$ & $\mathrm{~A} 1.82+\mathrm{AFOSC}+\mathrm{gm} 4$ & $\cdots$ \\
\hline 20020912 & 52529.88 & +5.7 & $3250-9800$ & $\mathrm{CA}+\mathrm{CAFOS}+\mathrm{B}, \mathrm{R} 20^{\dagger}$ & $\mathrm{BD}+284211$ \\
\hline 20020916 & 52533.84 & +9.6 & $3300-8700$ & $\mathrm{INT}+\mathrm{IDS}+\mathrm{R} 150 \mathrm{~V}$ & Kopff 27 \\
\hline 20020918 & 52535.03 & +10.8 & $3450-9600$ & D1.54+DFOSC+gm4 & LTT 7987 \\
\hline 20020919 & 52536.87 & +12.7 & $3450-8800$ & $\mathrm{INT}+\mathrm{IDS}+\mathrm{R} 150 \mathrm{~V}^{\dagger}$ & Kopff 27 \\
\hline 20020922 & 52540.00 & +15.8 & $3300-9750$ & D1.54+DFOSC+gm5 & LTT 7987 \\
\hline 20020923 & 52540.86 & +16.7 & $3300-9700$ & $\mathrm{INT}+\mathrm{IDS}+\mathrm{R} 150 \mathrm{~V}$ & Kopff 27 \\
\hline 20020924 & 52542.00 & +17.8 & 9400-24900 & NTT+SofI & HIP 95550 \\
\hline 20020926 & 52544.02 & +19.8 & $3500-8800$ & D1.54+DFOSC+gm5 & LTT 7987 \\
\hline 20021001 & 52548.75 & +24.6 & $3500-7500$ & $\mathrm{~A} 1.82+\mathrm{AFOSC}+\mathrm{gm} 4$ & $\mathrm{BD}+284211$ \\
\hline 20021010 & 52557.84 & +33.6 & $3600-9300$ & INT+IDS+R150V & Kopff 27 \\
\hline 20030410 & 52739.17 & +215.0 & $3500-8000$ & TNG+LRB & Feige 66 \\
\hline
\end{tabular}

Notes. ${ }^{*}$ Epoch relative to $B_{\max }$, which occurred on MJD $=52524.2$ (Pignata et al. 2004). A1.82 = Asiago $1.82 \mathrm{~m}$ telescope, CA = Calar Alto $2.2 \mathrm{~m}$ telescope, INT = Isaac Newton Telescope, D1.54 = Danish $1.54 \mathrm{~m}$ telescope, NTT $=$ ESO New Technology Telescope, TNG $=$ Telescopio Nazionale Galileo. For the $-8.3 \mathrm{~d}$ spectrum we used the flux standard taken with the same set-up on the previous night, while for the $+4.6 \mathrm{~d}$ spectrum we used a sensitivity function appropriate for the setup, kindly provided by N. Elias de la Rosa. $\dagger$ Data not flatfielded; $\ddagger$ missed target with blue grism.

spectra was apparent on several nights and variable conditions exacerbated the situation. To correct for this distortion we multiplied the fluxed supernova spectra by the $B V R I$ Bessell filter functions and integrated across the bands. A similar procedure was applied to the spectrum of Vega, and hence spectroscopic magnitudes for the supernova were derived. Comparison with near-contemporaneous photometry (from Pignata et al. 2004) was then used to derive scaling factors for each band. A loworder polynomial fit to the scaling factors was obtained which was then applied to full supernova spectrum. We estimate the flux calibration to be accurate to about $10-15 \%$. For the earliest epochs ( -11 to $-8 \mathrm{~d}$ ) no photometry was available for comparison. Since it is the behaviour at the earliest epochs that we seek to understand, we did not scale these spectra in any preconceived manner.

\section{Optical spectra at early times}

This section is subdivided as follows: we detail the early spectral evolution of SN 2002er in Sect. 3.1 followed by a discussion on the presence of carbon in Sect. 3.2. We then describe the evolution of the expansion velocities in Sect. 3.3.

\subsection{Spectral evolution}

The spectral evolution of SN 2002er from day -11 to +34 is shown in Fig. 2. The rapid evolution at early times can be followed in detail given the excellent temporal coverage. The outermost material in the ejecta, composed mainly of intermediate mass elements is responsible for producing the spectrum up to about maximum light. The spectra shown in Fig. 2 are typical of normal type Ia supernovae, characterised at early epochs (up to about day +6 ) by the deep P Cygni absorption near $6150 \AA$ due to SiII and the distinctive W-shaped SII feature near $5400 \AA$. Other prominent features at these phases are attributed to the CaII $\mathrm{H}$ and $\mathrm{K}$ blend, MgII $\lambda 4481 \AA$ and a multitude of blends due to FeII, SiII, and SII in the 4500 to $5000 \AA$ range. The NaI D lines are clearly visible suggesting that substantial reddening due to intervening material.

A comparison with other type Ia supernovae at four representative epochs is shown in Fig. 3. The decline-rate 


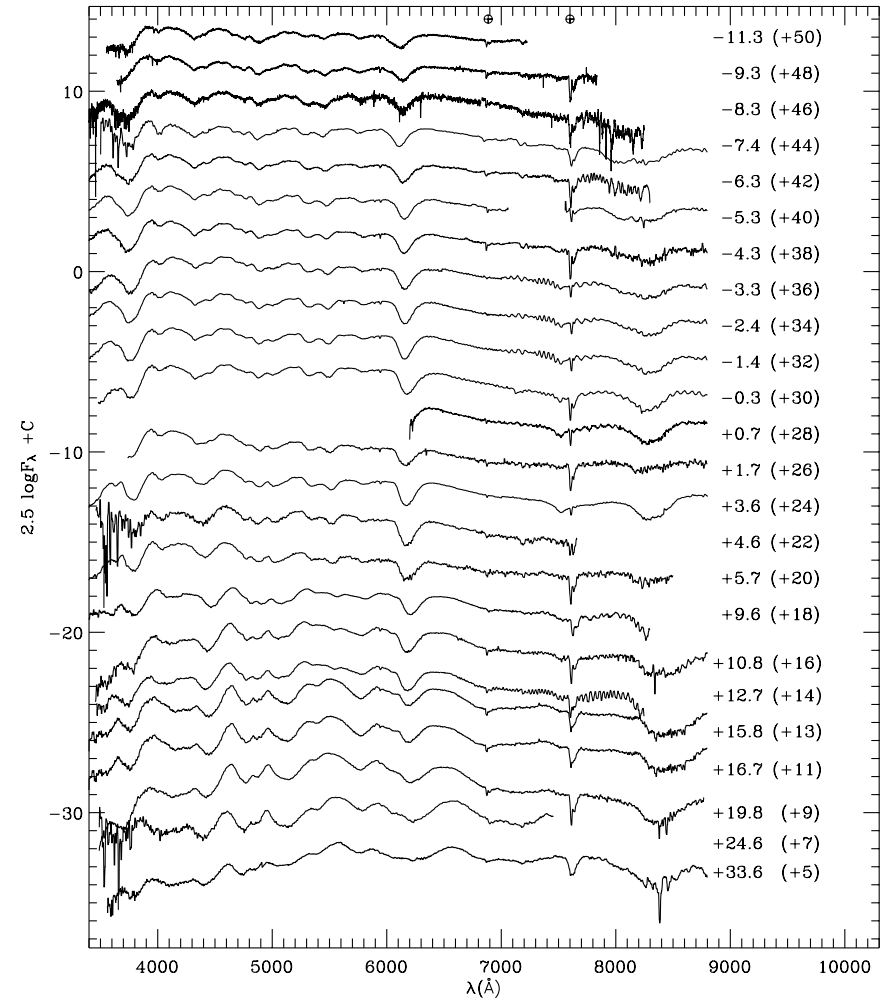

Fig. 2. Spectral evolution of SN 2002er during the photospheric phase. The spectra shown here have neither been corrected for the redshift of UGC 10743, nor have they been corrected for reddening. The spectra have been shifted vertically for clarity; these shifts are indicated in parenthesis next to the epoch of the spectra (see also Table 2). Note that the quality of the spectra beyond $\sim 9000 \AA$ is poor. The prominent telluric features are marked by earth symbols.

parameters for these supernovae are fairly typical with the exception of the slow-decliner SN 1999 ee which shows markedly different behaviour at early epochs, but has been included for comparison. Although differences between the spectra are present at all epochs, these are most pronounced in the premaximum spectra (Fig. 3a). The obvious contrasts are in the shape and depth of the prominent features and in the amount of structure in the spectrum. The latter is probably due in part to the differences in the velocities of the ejecta; this is especially true of SN 2002bo for which the higher velocities have probably smeared out the weaker features. However, the similarity between SN 2002er and SN 1994D, down to the weakest of features is striking. The strength of the temperaturesensitive SiIII $\lambda 4553,4568 \AA$ line suggests that SN 2002er and SN 1994D spectra are intermediate in temperature compared with the hotter SN 1998bu and SN 1998aq spectra and the cooler SN 2002bo spectrum. The W-shaped SII feature at $\lambda 5454,5460 \AA$ is already apparent at $-11 \mathrm{~d}$ in the SN 2002er spectrum whereas it only became apparent at $-2 \mathrm{~d}$ in the spectrum of SN 1999ee (Hamuy et al. 2002).

By maximum light (Fig. 3b), the CaII lines near $8500 \AA$ have become significantly stronger and the W-shaped SII $\lambda 5454,5640 \AA$ feature is very well-developed. The CaII H and $\mathrm{K}$ feature (blended with the SiII $\lambda 3858 \AA$ ) is more similar to that of SN 2002bo than to the double-dipped feature seen in
Table 3. $E(B-V)$ values for the $\mathrm{SNe}$ shown in Fig. 3.

\begin{tabular}{lll}
\hline \hline & $E(B-V)$ & Reference \\
\hline SN 1994D & $0.06 \pm 0.02$ & Patat et al. (1996) \\
SN 1996X & $0.1 \pm 0.03$ & Salvo et al. (2001) \\
SN 1998 aq & $\ldots$ & Branch et al. (2003) \\
SN 1998bu & $0.32 \pm 0.04$ & Hernandez et al. (2000) \\
SN 1999 ee & 0.32 & Hamuy et al. (2002) \\
SN 2002bo & $0.43 \pm 0.1$ & Benetti et al. (2004a) \\
\hline
\end{tabular}

Notes. The absence of narrow Na D absorption lines in SN 1998 aq (Ayani \& Yamaoka 1998) implies little reddening.

SNe 1994D, 1996X and 1998bu. Lentz et al. (2000) have suggested that the presence or absence of a split in this feature is related to the relative strength of the SiII and $\mathrm{Ca}$ features. The strength and width of the SiII $\lambda 6355 \AA$ feature is comparable to that seen in SN 1994D and SN 1996X. The $14950 \AA$ feature is stronger in SN 2002er at pre-maximum epochs than in the other supernovae shown in Fig. 3a; by maximum light, it has developed into a pronounced $\mathrm{W}$-shaped feature. Unlike the W-shaped SII feature at $\sim 5400 \AA$ which is ubiquitous at maximum in all normal type Ia supernovae, only in SN 1996X does the $\lambda 4950 \AA$ feature appear with comparable prominence to that of SN 2002er. The higher temperature of SN 2002er, as suggested by the SiIII lines, lends credence to the suggestion that the $24950 \AA$ feature is due to FeIII (Mazzali et al. 1995). Note that the presence of lines of high excitation is to be expected given the somewhat higher than normal ${ }^{56} \mathrm{Ni}$ mass inferred from the light curve by $\left(0.7 M_{\odot}\right.$ Pignata et al. 2004).

Nugent et al. (1995) found a tight correlation between the ratio, $\mathcal{R}(\mathrm{SiII})$ of the depths of the SiII $\lambda 5972 \AA$ and $\lambda 6355 \AA$ absorption troughs near maximum light and the luminosity of the supernova: the subluminous, faster decliners have larger values of $\mathcal{R}(\mathrm{SiII})$. However, this correlation has been shown to break down for $\Delta m_{15}(B) \lesssim 1.2$ (see Fig. 8 in Benetti et al. 2004a). For SN 2002er we measure 0.23 for $\mathcal{R}$ (SiII) which is consistent with the Nugent/Benetti relation to within the errors.

By about $+20 \mathrm{~d}$ post maximum light, the SiII $\lambda 6355 \AA$ feature is still clearly visible (Fig. 3d). All the other supernovae, shown at comparable epochs, show kinks in the red wing of the SiII $\lambda 6355 \AA$ feature due to FeII. SN 2002er, on the other hand, shows a rather smooth red wing. This suggests that the Sill feature is not confined to a narrow range in velocity and corroborates the finding by Pignata et al. (2004) from light curve modelling, that the ejecta are highly mixed. Notice also that the strength of the CaII $\mathrm{H}$ and $\mathrm{K}$ feature is more similar to that of the slowest decliner shown in Fig. 3, SN 1999ee. By about a month post-maximum light (Fig. 3e), the SilI feature is barely perceptible as features due to $\mathrm{Fe}$ dominate this wavelength range. Figures $3 d$ and e together suggest that the intermediate-element phase was of slightly longer duration in SN 2002er (i.e. slower transition to Fe-peak elements) than for the other supernovae shown in Fig. 3.

Interestingly, although the similarity of SN 2002er with SN 1994D and SN 1996X remains impressive throughout its 

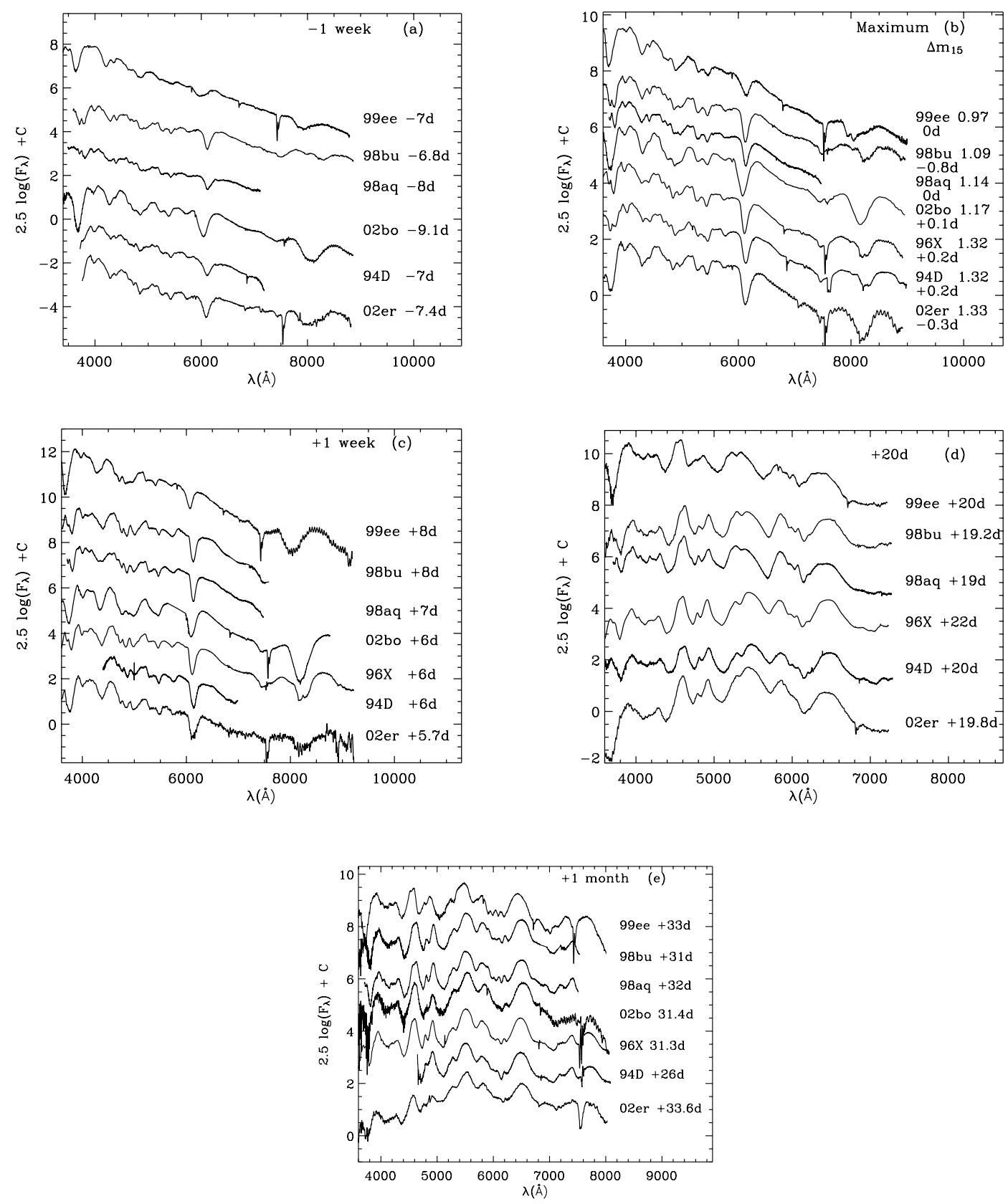

Fig. 3. Spectra of SN 2002er compared with other type Ia SNe at four representative epochs. The spectra have been shifted vertically by arbitrary amounts and have been corrected for the respective redshifts of the host galaxies. All spectra (except those of SN 1998aq) have been corrected for extinction using the values in Table 3 and the relation of Cardelli et al. (1989). The $\Delta m_{15}$ values shown in panel b) have been corrected for reddening following Phillips et al. (1999). Some of the principal spectral features at early epochs are marked in panel a).

photospheric evolution, the differences become more apparent with time.

\subsection{Carbon in pre-maximum spectra}

Carbon is a diagnostically interesting element in pre-maximum spectra. Its abundance allows us to place limits on the composition of the progenitor white dwarf. In addition, the amount of carbon constrains the extent of burning in the outer layers thereby testing various explosion scenarios. For instance, current delayed-detonation models have very little carbon below about $30000 \mathrm{~km} \mathrm{~s}^{-1}$, while the 1D deflagration model (W7) has unburnt carbon present down to velocities of about $14000 \mathrm{~km} \mathrm{~s}^{-1}$ (e.g. Branch et al. 2003).

The first reported detection of carbon in a SN Ia was in the -14 d spectrum of SN 1990N (Fisher et al. 1997). However, carbon lines have also been identified at $-10 \mathrm{~d}$ in the spectra of SN 1994D and at $-9 d$ in SN 1998aq (Branch et al. 2003). The strongest line is at $\lambda 6580 \AA$ with weaker lines at $\lambda 4267 \AA$ and $\lambda 7234 \AA$ A. In Fig. 4, we show tentative evidence for the presence of the CII $\lambda 6580 \AA$ Aine present up to about a week before maximum light at about $13000 \mathrm{~km} \mathrm{~s}^{-1}$. There is no evidence of 

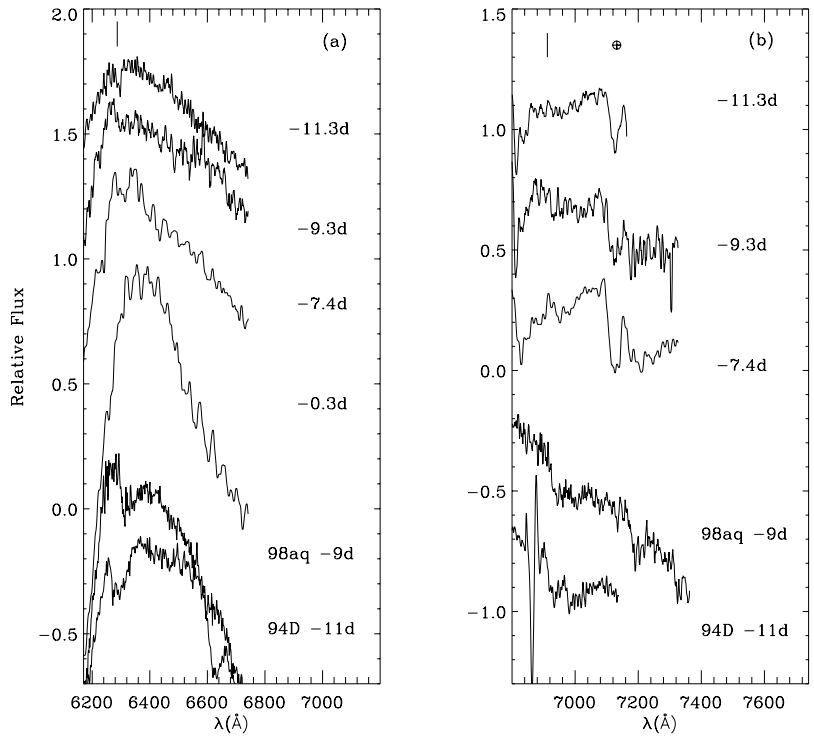

Fig. 4. Expanded view of several epochs of pre-maximum spectra around the CII $\lambda 6580,7234 \AA$ lines. The 02er spectra have been smoothed and shifted vertically for clarity. a) A short vertical dash shows the position of the weak feature seen in the earliest spectra. b) The dash shows the location of the $\lambda 7234 \AA$ line at the velocity $\left(13000 \mathrm{~km} \mathrm{~s}^{-1}\right)$ inferred from panel a). The earliest spectra of SN 1994D and SN 1998aq are shown for comparison.

the CII $\lambda 7234 \AA$ line (Fig. 4b) but this would, in any case, be expected to be much weaker.

\subsection{Expansion velocities}

As the photosphere recedes into deeper and slower moving material, the minima of the absorption features shift redwards with time. As already mentioned, this can provide a powerful "tomographic" picture of the SN ejecta. However, very strong lines may be less useful in this regard. Patat et al. (1996) point out that the velocities inferred from the strong features (e.g. the characteristic SiII line) are only representative of the photospheric velocity at early phases when the amount of material above the photosphere is small; as the photosphere retreats, it encounters flatter density gradients which results in strong lines being formed over a large velocity range, thus overestimating the photospheric velocity. In contrast, weak lines can provide good indicators of the photospheric position.

In SN 2002er, we examined the velocity evolution of the strong SiII $\lambda 6355 \AA$ and CaII $\mathrm{H}$ and $\mathrm{K}$ lines and the weak SII $\lambda 5640 \AA$ line. The results are shown in Fig. 5 together with those of other supernovae for comparison. All velocities have been corrected for the recession velocities of the respective host galaxies. As expected, the weak SII line exhibits lower velocities than do stronger lines. The strong SiII feature in the earliest spectrum of SN 2002er $(-11.3 \mathrm{~d})$, has its minimum centred at about $6120 \AA$, with its blue edge lying at about $22000 \mathrm{~km} \mathrm{~s}^{-1}$. As has already been pointed out by several authors (e.g. Mazzali et al. 1993) such velocities are well in excess of the $15000 \mathrm{~km} \mathrm{~s}^{-1}$ limit in the unmixed $\mathrm{W} 7$ model (Nomoto et al. 1984).

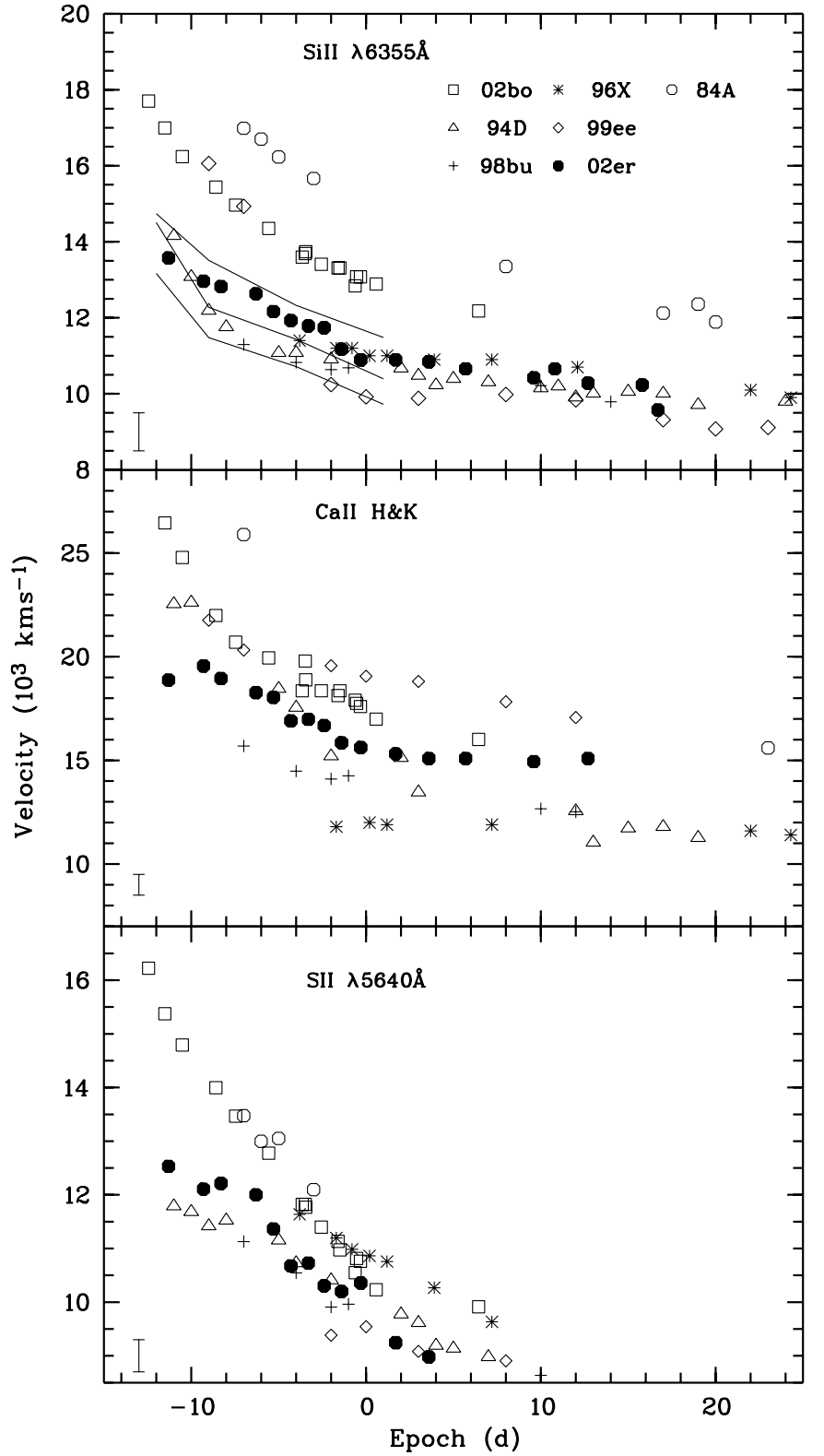

Fig. 5. The velocity evolution of the SiII $\lambda 6355 \AA$, Ca H and K, and SII $\lambda 5640 \AA$ features compared with other $\mathrm{SNe}$ as measured from the minima of the respective absorption features. The solid curves shown in the top panel are models of different metallicity (top: $\times 10$; middle: $\times 1$; bottom: $\times 1 / 10$ solar) obtained from Lentz et al. (2000). Typical error bars for SN 2002er are shown in the bottom left-hand corner of each panel.

Lentz et al. (2000) have computed the effects of varying the metallicity in the $\mathrm{C}+\mathrm{O}$ layer of the standard deflagration model, W7, on the emergent pre-maximum spectra of type Ia supernovae. They find that the strength, profile, and velocity of the SiII $\lambda 6355 \AA$ feature is a function of metallicity. Their results are plotted in Fig. 5. Clearly the range of velocities seen between a number of SNe Ia may indeed be due to metallicity variations. However, even with implausibly large variations in the $\mathrm{C}+\mathrm{O}$ layer metallicity $(\times 1 / 10$ to $\times 10$ solar $)$ we cannot reproduce the high velocities exhibited by SN 2002bo and 
SN 1984A. These large velocities could be due to other factors such as higher explosion energy.

\section{Synthetic spectra}

Comparison with observations of synthetic spectra based on specific explosion models provides a powerful tool for probing the physics of supernovae. Here we compare the SN 2002er early-time spectra with model spectra generated by a Monte Carlo (MC) code. The code is based on the Sobolev approximation, and it was discussed in a number of papers that the reader can turn to for details (Abbott \& Lucy 1985; Mazzali \& Lucy 1993; Lucy 1999; Mazzali 2000). The input parameters are the luminosity $L$, the epoch $t_{\exp }$ where explosion occurs at $t_{\text {exp }}=0 \mathrm{~d}$, the photospheric velocity $v_{\mathrm{ph}}$, and a density distribution from an explosion model. The code iteratively computes the radiation field and the level populations in the SN ejecta under the assumption that there is no net exchange of energy between matter and radiation above a sharply defined photosphere. This means that no deposition of fast particles created in radioactive decays occurs above the photosphere, and that absorptive continuum opacities are ignored. This is a reasonable approximation at the earliest phases of a SN Ia.

The luminosity is chosen to match the observed value and the velocity adjusted to obtain a good match to the observed line blueshifts. The epoch relative to explosion is determined, inevitably with some uncertainty, from the time of observed maximum. This is because first observations are usually at least a few days after the SN exploded. Different models can be chosen for the density distribution. In this paper we base our calculations on W7 (Nomoto et al. 1984), which is known to give a good description of typical type Ia SNe. Energy packets are released at the photosphere and travel through the SN envelope, where they can undergo line absorption and reemission (branching) and electron scattering. When a converged temperature structure is established and appropriate excitation and ionisation conditions are computed (using a modified nebular approximation (Abbott \& Lucy 1985; Mazzali \& Lucy 1993), a synthetic spectrum is obtained using a formal integral scheme.

We calculated two spectral models for SN 2002er, one for an epoch near maximum light (day -2.4) and one for the earliest epoch at -11.3 days. These two epochs were chosen to cover a period in which the physical properties change significantly.

In the first iteration of the day -2.4 spectral model we adopted a typical value for the photospheric velocity at this epoch, of $9500 \mathrm{~km} \mathrm{~s}^{-1}$. The distance modulus $(\mu=32.9)$ and the reddening $(E(B-V)=0.36)$ were taken from Pignata et al. (2004). This initial set of parameters provided a poor match to the observed spectrum. The problem was that, in order to match the observed bolometric luminosity of $\log _{10} L \approx 43.2\left[\mathrm{erg} \mathrm{s}^{-1}\right.$ ] (Pignata et al. 2004) and the observed line velocity (this work) the model temperature near the photosphere had the rather high value of $T_{\mathrm{BB}} \approx 14000 \mathrm{~K}$. Consequently the ionisation was much too high. For example, strong Si III lines were predicted but not observed. The large observed line ratio of Si II $5970 \AA$ to Si II $6350 \AA$ also indicates a rather low temperature.
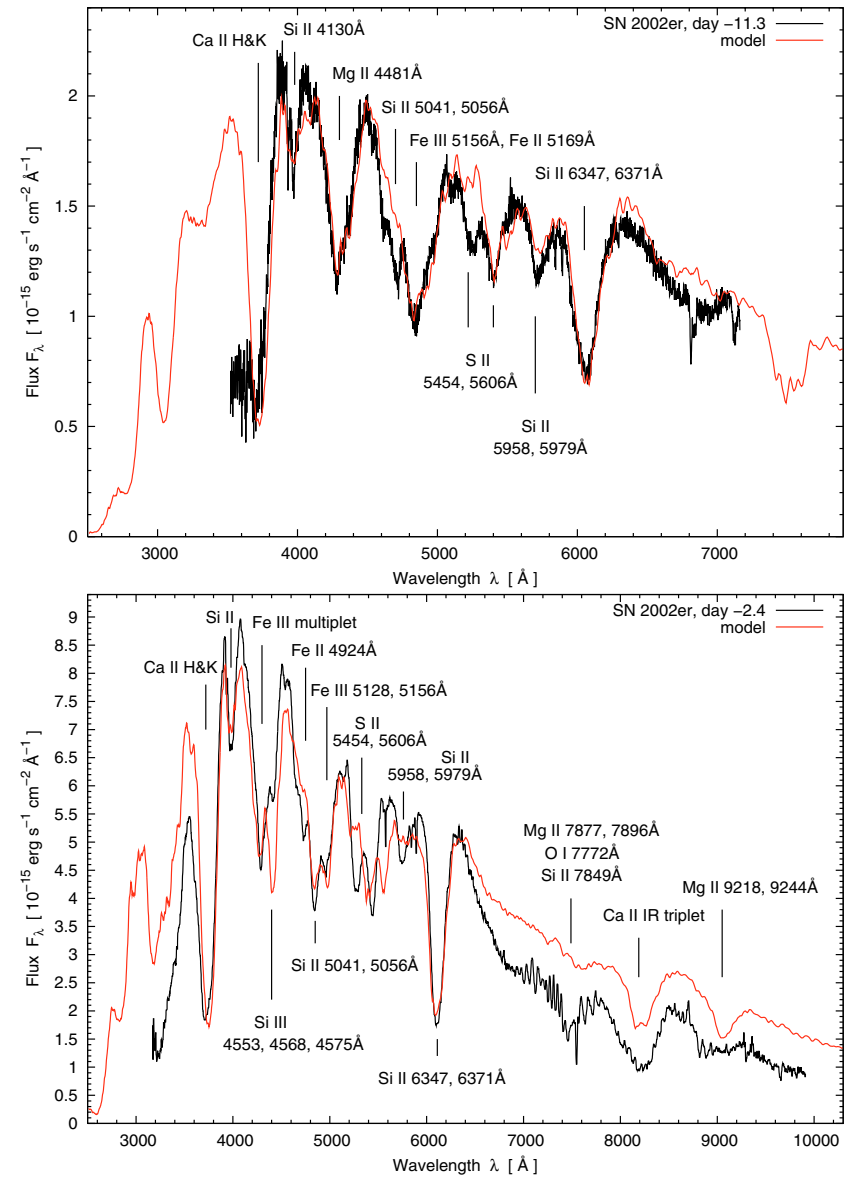

Fig. 6. Observed spectrum and corresponding model of SN 2002er at using $E(B-V)=0.36$ and $\mu=32.70$ with $t_{\exp }=8.7 \mathrm{~d}$ (top panel) and $t_{\text {exp }}=17.6 \mathrm{~d}$ (bottom panel).

Nugent et al. (1995) derived a temperature of $\sim 9000 \mathrm{~K}$ for SNe Ia with a line ratio similar to that of SN 2002er.

In order to try to resolve this discrepancy between the model and observations, we explored the range of parameter space available through the uncertainties in reddening, distance and explosion epoch. We found that even if we set the reddening at the $1 \sigma$ lower limit of $E(B-V)=0.31$ (Pignata et al. 2004), we make little difference to the discrepancy. The best match was obtained by pushing the distance modulus to its $1 \sigma$ lower limit of $\mu=32.7$, while keeping the formal value of the reddening, $E(B-V)=0.36$, and using a risetime of $20.0 \mathrm{~d}$. This risetime is $1.3 \mathrm{~d}$ longer than estimated by Pignata et al. (2004), but is closer to the typical SN Ia risetime of $t_{B_{\max }}=19.5 \mathrm{~d}$ (Riess et al. 1999). This departure from the Pignata et al. (2004) value can be justified by the fact that the photometric data are not available earlier than -7.3 days. Nevertheless, even with these more extreme parameter values, the temperature was still found to be rather too high. The best match parameters are $\log _{10} L=43.15\left[\mathrm{erg} \mathrm{s}^{-1}\right]$ and a velocity at photophere of $9200 \mathrm{~km} \mathrm{~s}^{-1}$, leading to a photospheric temperature of $T_{\mathrm{BB}}=12460 \mathrm{~K}$. The model thus obtained is compared with the observed spectrum in Fig. $6 \mathrm{~b}$.

The main features are labelled with their rest wavelength in Fig. 6. We identify strong Si II lines at $6360 \AA$, $5970 \AA$, and at 
$4130 \AA$ Ad Aditionally, the deep absorption near $4800 \AA$ A contains a significant amount of Si II. The rest of this feature is caused by Fe II. Weak Si III lines can be seen in the observed spectrum at $4570 \AA$ and $5740 \AA$, but they are both very strong in the model due to the high temperature. S II produces the double line feature near $5400 \AA$. The absorption at $\sim 4400 \AA$ is due to $\mathrm{Mg}_{\text {II }} 4481 \AA$, as well as the one near $7500 \AA$. Finally we recognise the strong $\mathrm{Ca}$ II $\mathrm{H}$ and $\mathrm{K}$ lines and the Ca II IR triplet. Although it is not as pronounced as in the case of SN 2002bo (Stehle et al. 2004), a high-velocity component in the Ca II lines can be seen that is not reproduced by the model, which uses the density distribution of W7.

In constructing the spectral model for -11.3 days, we could not obtain the flux directly from observation since the earliest photometry epoch was at -7.3 days. One possibility is to extrapolate the light curve to the early epochs using a quadratic function in time (Riess et al. 1999) ( $\left.L \propto t_{\mathrm{exp}}^{2}\right)$. This procedure gives $\log _{10} L \simeq 42.75$ [erg s${ }^{-1}$ ]. This is however too bright, leading to a very hot model. An alternative approach is to used the synthetic light curve fit of Pignata et al. (2004) to extrapolate back to -11.3 days. This yielded $\log _{10} L \simeq 42.6\left[\mathrm{erg} \mathrm{s}^{-1}\right.$ ]. This allowed the model to produce much more reasonable results. The best match was obtained with a velocity at the photosphere of $13000 \mathrm{~km} \mathrm{~s}^{-1}$, leading to $T_{\mathrm{BB}}=10950 \mathrm{~K}$. The model spectrum is compared with the observed spectrum in Fig. 6 . Although the agreement is good, it should be kept in mind that the synthetic light curve shape may change when a more realistic element distribution is adopted compared with that used in Pignata et al. (2004). It is interesting that in SN 2002er, as in SN 2002bo, the temperature rises as we approach maximum light. This behaviour is not typical of all SNe Ia (Benetti et al. 2004b)

While the strong Si II line absorptions are well reproduced, Si II $5970 \AA$ is too weak owing to the temperature still being too high. The $\mathrm{S}$ II double line feature near $5400 \AA$ is not well reproduced in its blue part. Since these lines come from the same lower levels, there may be a problem in the model line list, in particular the $g f$ values. The line at $\sim 4700 \AA$ is not reproduced by the model. This feature was detected in other SNe Ia and can possibly be assigned to a high-velocity absorption of Fe II (Hatano et al. 1999). All the other lines are well reproduced and have already been discussed for the day -2.4 model.

The above analysis indicates shortcomings in the modelling procedure. Specifically, owing to the homogenous abundance distribution of the model, to reproduce the strong Si II absorptions we had to introduce a rather high $\mathrm{Si}$ abundance (63\% by mass) throughout the envelope. But due to the high photospheric temperature, the $\mathrm{Si}$ is almost completely doublyionised near the photosphere at maximum, severely overproducing the Si III features. Only further out in the envelope, where the temperature is sufficiently low, are the required Si II absorptions formed. A similar problem was encountered with SN 2002bo (Benetti et al. 2004a). However, Stehle et al. (2004) find that, in a more recent analysis of SN 2002bo by the "Abundance Tomography" method, it is possible to reduce the Si abundance near the photosphere and yet simultaneously keep a desirably high $\mathrm{Si}$ abundance at the outer radii
Stehle et al. (2004). Furthermore, Stehle et al. (2004) also find that improving the description of the radiation field at the inner boundary reduces the overestimate of the emergent flux in the red, leading to a decrease of the total luminosity and hence a lower temperature near the photosphere. In the case of SN 2002er, however, we also note that if the reddening and/or the distance are reduced by more than the $1 \sigma$ error boundaries estimated by Pignata et al. (2004) then greater consistency with the spectral models is achieved. At present, there is no independent evidence that such reduction should be applied.

We conclude that the maximum light spectrum of SN 2002er can be fairly well modelled using a homogeneous abundance distribution in the atmosphere provided we push the adopted distance and risetime close to the observational limits. Modelling of the earliest spectrum depends sensitively on the adopted flux level, but unfortunately observations do not directly provide this value. However, extrapolation by means of a bolometric light curve model fit appears to provide a reasonable estimate of this quantity. Future progress will require a more realistic model which includes a stratified abundance distribution.

\section{Near-IR spectroscopy of SN 2002er}

Early-time IR spectroscopic observations can provide a particularly powerful diagnostic of the dynamic model because they probe different depths at the same epoch within the exploded white dwarf through the strongly variable lineblanketing opacity (Wheeler et al. 1998). Furthermore, the large number of lines in the optical/UV regions, coupled with the strong Doppler-broadening (several thousand $\mathrm{km} \mathrm{s}^{-1}$ ) can make species identification difficult. Strong extinction effects often introduce additional error. In contrast, the near-IR has fewer lines and reduced sensitivity to extinction uncertainty, allowing firm line identification and accurate measurement of line strength and evolution. In addition, since there are much fewer strong resonance lines, it means that, in general, for a line to be detectable it needs a significant element abundance. In other words, IR lines suffer less from contamination by primordial elements.

After maximum light there is a rich mixture of Fe-group lines in the IR spectrum, allowing us to explore the innermost regions of the supernova and to ascertain the extent of incomplete silicon burning. Wheeler et al. (1998) find that the post-maximum-light features at $\sim 1.5-1.7$ and $2.2-2.6 \mu \mathrm{m}$ are primarily due to iron group elements of the second ionisation stage. The blue extent of the feature at $\sim 1.55 \mu \mathrm{m}$ defines the transition from partial to complete Si burning (Marion et al. 2003). Due to the high optical depth, this feature is formed near the outer boundary of the $\mathrm{Fe} / \mathrm{Co} / \mathrm{Ni}$ core and demarcates the Fe/Si edge (Fig. 7) Wheeler et al. (1998) particularly note that the minimum at $\sim 1.7 \mu \mathrm{m}$ provides a diagnostic tool for assessing the velocity spread of the region containing radioactive materials which, in turn, depends on the underlying explosion scenario. The $2.2-2.6 \mu \mathrm{m}$ features also contain significant contributions from intermediate mass elements such as $\mathrm{Si}$. Indeed, these $K$-band features are formed in the transition region between complete and incomplete silicon burning and are 


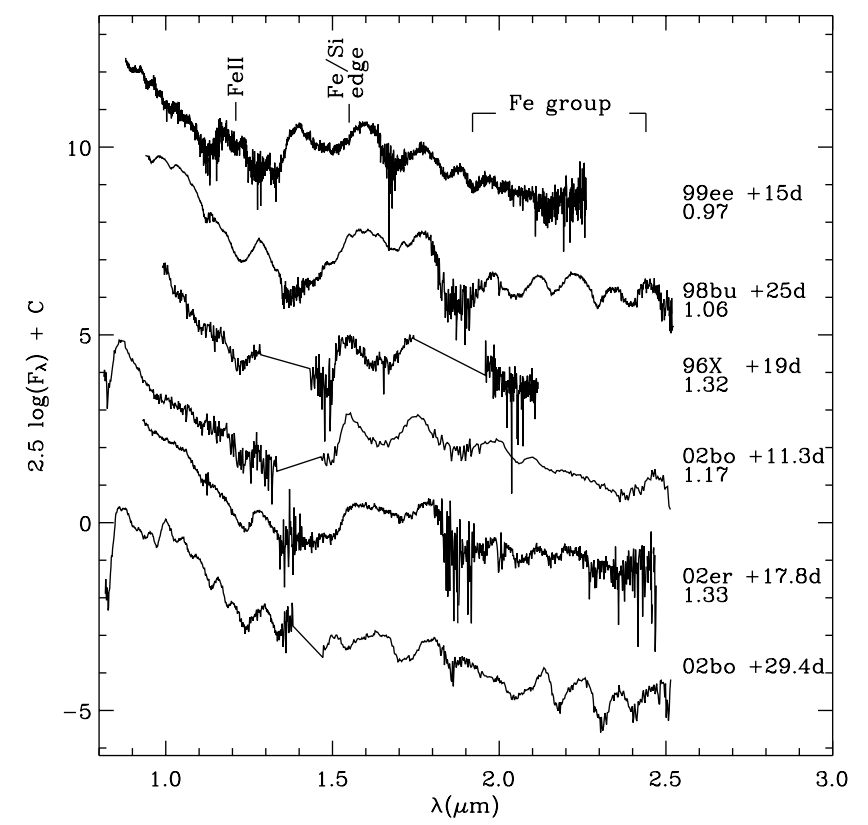

Fig. 7. Near-infrared spectrum of SN 2002er at +18 d compared with spectra of other SNe Ia at comparable epochs. The spectra of SNe 1998bu, 1996X, 1999ee, and 2002bo have been taken from Jha et al. (1999), Hernandez et al. (2000), Hamuy et al. (2002), and Benetti et al. (2004a) respectively. The spectra are shown in the respective rest-frames of the supernovae and have been de-reddened using the values in Table 3. The epochs relative to maximum light are shown next to the name and the $\Delta m_{15}$ values are noted just below. Prominent features are indicated following Marion et al. (2003).

sensitive to the composition structure and hence the explosion model.

Due to a combination of poor conditions and instrument availability, only a single epoch of near-IR spectroscopy was obtained, on 2002 September 24 (+17.8 d) using the NTT/SofI low-resolution blue and red grisms. The spectrum was reduced using standard procedures in the FIGARO 4 environment. Wavelength calibration of the 0.95-1.64 $\mu \mathrm{m}$ spectrum was carried out using a $\mathrm{Xe}$ arc; as no arc frame was available for the 1.53-2.52 $\mu \mathrm{m}$ spectrum, we used the $\mathrm{OH}$ sky lines. Flux calibration was carried out with respect to the bright F6V star HIP 95550 and compared with near-IR photometry taken at $+19.9 \mathrm{~d}$ (Pignata et al. 2004). The spectrum, scaled by $20 \%$ to match the photometry, is shown in Fig. 7. For comparison, we also show the rest-frame near-IR spectra of other SNe Ia at comparable epochs. Owing to the sparsity of near-IR spectra at the appropriate epoch ( $\sim 3$ weeks), we include two spectra of SN 2002bo that bracket the phase of the SN 2002er near-IR observation.

Inspection of Fig. 7 shows that the IR spectrum of SN 2002er is fairly typical for the epoch considered. As anticipated for this epoch, the spectrum is dominated by the increasingly exposed Fe-group elements. The $1.7 \mu \mathrm{m}$ feature is considerably shallower in SN 2002er than in SN 1996X and is more reminiscent of the morphology seen in the somewhat faster decliners such as SN 1998bu and perhaps, SN 1999ee. As indicated above, this may be interpreted as a larger-thanaverage velocity spread in the radioactive materials. However, the measured Doppler blueshift in the $1.5 \mu \mathrm{m}$ edge (rest wavelength $1.57 \mu \mathrm{m}$, Marion et al. 2003) is $8300 \pm 500 \mathrm{~km} \mathrm{~s}^{-1}$ for SN 2002er compared with $10500 \pm 500 \mathrm{~km} \mathrm{~s}^{-1}$ for SN 1996X at $+19 \mathrm{~d}$. This suggests that silicon burning actually extended to higher velocities in SN 1996X.

\section{The nebular spectrum at +215 days}

In a type Ia supernova, by about 60-100 days post-explosion the photosphere has receded to the centre, and the ejecta have become transparent to the optical/IR emission i.e. the forbidden-line-dominated nebular phase has been reached. The transparency of the ejecta means that the physical conditions and constituents of the innermost regions can be directly probed. Furthermore homologous expansion means that line profiles provide valuable information about the distribution of the nucleosynthesised materials. In particular, the mass and velocity distribution of ${ }^{56} \mathrm{Ni}$ and its products can provide information on the nature of the explosion.

We obtained a single nebular phase spectrum at $+215 \mathrm{~d}$ post $B$-maximum. The spectrum was reduced as described in Sect. 2 and is shown in Fig. 8 together with spectra of other type Ia supernova at comparable epochs. As with the earlier epoch spectra, the form of the late time spectrum of SN 2002er is not unusual. The spectrum is dominated by forbidden lines of singly and doubly ionised Co and Fe. The FWHM of the $4700 \AA$ feature $\left(\sim 13000 \mathrm{~km} \mathrm{~s}^{-1}\right)$ is consistent with the relation in Mazzali et al. (1998) that links the width of nebular lines to the luminosity of the supernova.

In order to exploit the information available in the nebular spectrum, we compared it with the non-LTE nebular spectral model described in Bowers et al. (1997), to which we refer the reader for details. Briefly, the model comprises a uniform density, homologous expanding sphere containing iron, cobalt, and sulphur with the relative abundances as specified by radioactive decay. Forbidden lines of these elements emitted by singly and doubly-ionised species are predicted to dominate the nebular phase at optical and near-IR wavelengths. Consequently only these two ionisation stages were included in the model. The distance and reddening were set at $\mu=32.9$ and $E(B-V)=0.36$ respectively (see above). The ${ }^{56} \mathrm{Ni}$ mass, expansion velocity, ionisation degree and temperature are free parameters. The best-match model is shown in Fig. 9, compared with the observed spectrum. The model parameter values are $T=6600 \mathrm{~K}$, expansion velocity $\sim 9000 \mathrm{~km} \mathrm{~s}^{-1}$ fractional abundance of SII = 0.5 , and $M\left({ }^{56} \mathrm{Ni}\right)=0.69 M_{\odot}$. These clearly yield a fair match to the observed spectrum. We also note that the derived mass of ${ }^{56} \mathrm{Ni}$ is close to the value obtained from the bolometric light curve $\left(0.7 M_{\odot}\right.$, Pignata et al. 2004). We conclude that (i) the masses of ${ }^{56} \mathrm{Ni}$ derived by the two methods are consistent with each other, and (ii) the fraction of neutral and triply-ionised iron-group elements is indeed quite small.

\section{Summary}

We have presented extensive optical spectroscopy of SN 2002er at epochs spanning -11 to +215 days. The spectroscopic coverage at epochs up to +34 days is especially 


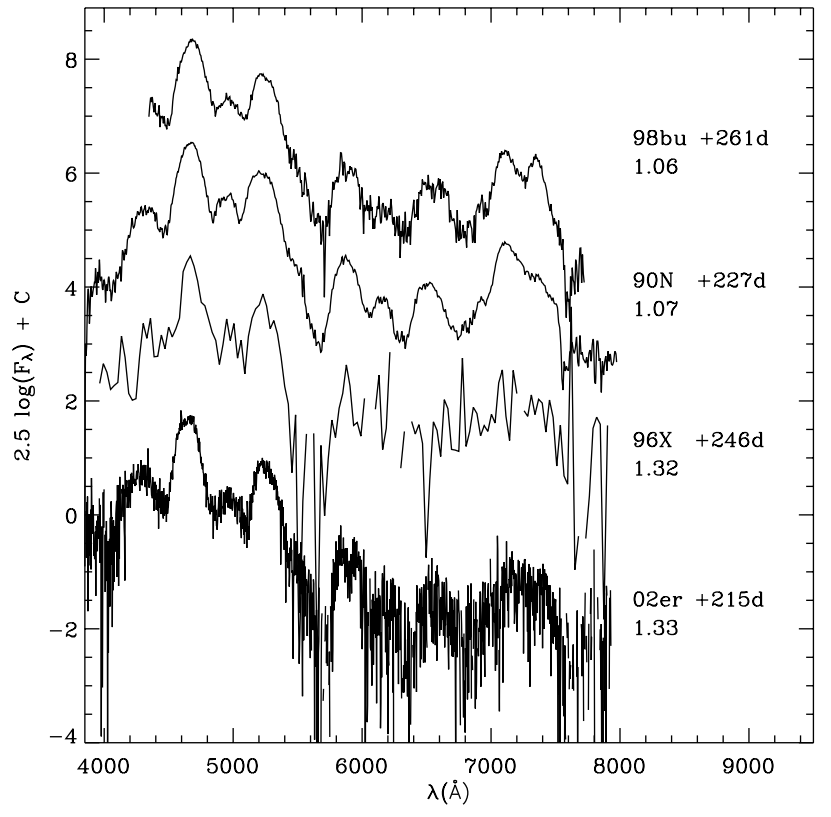

Fig. 8. The nebular spectrum of SN 2002er compared with the few spectra that are available of other SNe Ia at comparable epochs. The epochs relative to maximum light are indicated next to the names and the $\Delta m_{15}$ values are indicated just below. The spectra have been corrected for the redshift of their respective host galaxies and have been dereddened in the same manner as for Fig. 3. $F_{\lambda}$ is in units of $10^{-16} \mathrm{erg} \mathrm{cm}^{-1} \mathrm{~s}^{-1} \AA^{-1}$. The SN 1998bu, SN 1990N, and SN 1996X spectra have been shifted by $+5.2,+4.0$, and +3.2 respectively. The SN 1996X spectrum has been binned by a factor of 10 in an attempt to compensate for the very low signal in the 6000-7000 A region.

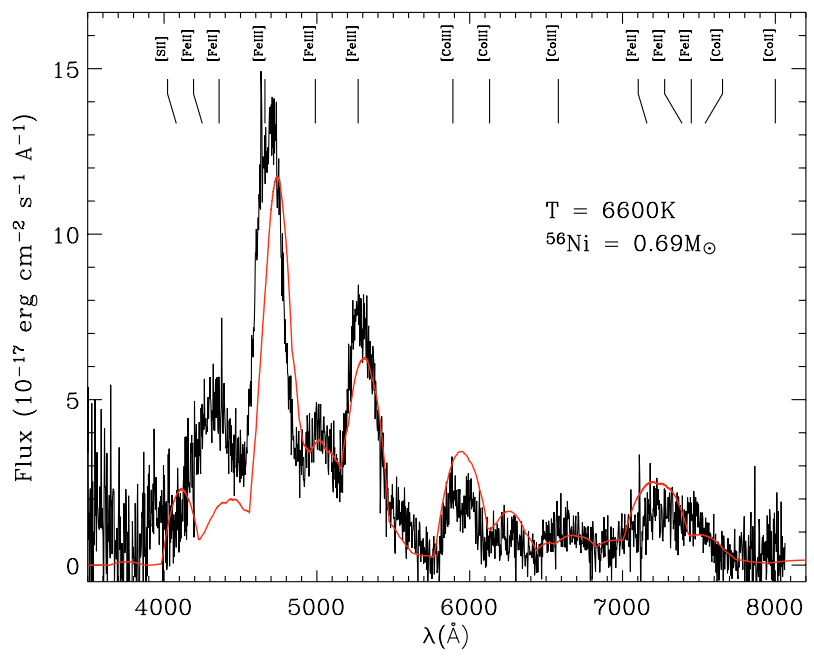

Fig. 9. The nebular spectrum of SN 2002er overlaid with a one-zone model. The model has been redshifted and reddened using the recession velocity of the host galaxy and $E(B-V)=0.36$. The main features have been labelled; note that most of these features are blends of several lines.

noteworthy. In most respects, SN 2002er bears the hallmarks of being a typical, albeit significantly reddened, type Ia supernova. Its spectral evolution was similar to those of other normal type Ia SNe such as SN 1994D and SN 1996X that suffered from minimal reddening. Nevertheless, differences are seen between the coeval spectra of SN 2002er and other normal $\mathrm{SNe}$ Ia. These are most pronounced in the pre-maximum spectra.

We compared the spectra at two early-time epochs with a homogeneous spectral synthesis model based on the deflagration model W7. The best match was obtained only by pushing the distance modulus to its $1 \sigma$ lower limit of $\mu=32.7$, and using a risetime as high as $20.0 \mathrm{~d}$. Even then the model temperature is rather too high. A similar problem was encountered at both epochs. Nevertheless, most of the features of the early-time spectra were quite successfully reproduced by the model. Future modelling improvements will include stratified abundances, plus a better description of the radiation field.

Only one early-time IR spectrum was obtained. As with the optical spectra, this showed that SN 2002er was fairly typical but with some differences from other events.

An optical spectrum obtained at $+215 \mathrm{~d}$ spectrum was modelled using a simple one-zone nebular code. This provided a fair match to the observed spectrum. A ${ }^{56} \mathrm{Ni}$ mass of $0.69 M_{\odot}$ was inferred - consistent with that derived from the light curve $\left(0.7 M_{\odot}\right.$ Pignata et al. 2004), and indicating that the fraction of neutral and triply-ionised iron-group elements is quite small.

The principal contribution of this paper to the study of the SN Ia phenomenon has been its provision of an extensive, frequently-sampled set of early-time spectra. These spectra span the crucial phase during which the photosphere recedes from the outermost unburned layers into the innermost iron-group regions of the core. This provides a superb dataset against which explosion scenarios will be tested via "tomographic" spectral synthesis models. Such studies will be vital in order to understand the significance of the small differences seen between different type Ia supernovae.

Acknowledgements. We are grateful to all the observers who had to give up part of their observing time so that SN 2002er could be observed, and to the staff at the various observatories for efficiently scheduling/carrying out our service/override requests. R.K. would additionally like to thank U. Thiele for remeasuring the arcs used in the CAFOS observations. We also appreciate A. Pastorello's help in acquiring the AFOSC observations. Support from the EC Programme "The Physics of type Ia SNe" (HPRN-CT-2002-00303) is acknowledged. This work is based on observations collected at the European Southern Observatory, Chile (ESO No. 69.D-0670), the Italian Telescopio Nazionale Galileo (TNG), La Palma, and the Isaac Newton (INT), and William Herschel (WHT) Telescopes of the Isaac Newton Group, La Palma, The TNG is operated on the island of La Palma by the Centro Galileo Galilei of INAF (Instituto Nazionale di Astrofisica) at the Spanish Observatorio del Roque de los Muchachos of the Instituto de Astrofisica de Canarias. The INT and WHT are operated on the island of La Palma by the Isaac Newton Group (ING) in the Spanish Observatorio del Roque de los Muchachos of the Instituto de Astrofisica de Canarias. Also based in part on observations collected at the CAHA at Calar Alto, operated jointly by the Max-Planck Institut für Astronomie and the Instituto de Astrofisica de Andalucia (CSIC). 


\section{References}

Abbott, D. C., \& Lucy, L. B. 1985, ApJ, 288, 679

Ayani, K., \& Yamaoka, H. 1998, IAUC, 6878

Benetti, S., Meikle, P., Stehle, M., et al. 2004a, MNRAS, 348, 261

Benetti, S., et al. 2004b, ApJL, submitted

Bowers, E. J. C., Meikle, W. P. S., Geballe, T. R., et al. 1997, MNRAS, 290, 663

Branch, D., Livio, M., Yungelson, L. R., et al. 1995, PASP, 107, 1019

Branch, D., Garnavich, P., Matheson, T., et al. 2003, AJ, 126, 1489

Cardelli, J. A., Clayton, G. C., \& Mathis, J. S. 1989, ApJ, 345, 245

Drell, P. S., Loredo, T. J., \& Wasserman, I. 2000, ApJ, 530, 593

Riess, A. G., Filippenko, A. V., Li, W., et al. 1999, AJ, 118, 2675

Fisher, A., Branch, D., Nugent, P., \& Baron, E. 1997, ApJ, 481, L89

Hamuy, M., Maza, J., Pinto, P. A., et al. 2002, ApJ, 124, 417

Hamuy, M., Phillips, M. M., Suntzeff, N. B., et al. 2003, Nature, 424, 651

Hatano, K., Branch, D., Fisher, A., et al. 1999, ApJ, 525, 881

Hernandez, M., Meikle, W. P. S., Aparicio, A., et al. 2000, MNRAS, 319,223

Hillebrandt, \& Niemeyer 2000, ARA\&A, 38, 191

Jha, S., Garnavich, P., Kirshner, R., et al. 1999, ApJS, 125, 73

Khokhlov, A. M. 1991, A\&A, 245, 114

Kotak, R., Meikle, W. P. S., Adamson, A., \& Leggett, S. K. 2004, MNRAS, 354, L13

Leibundgut, B. 2000, A\&AR, 10, 179

Lentz, E. J., Baron, E., Branch, D., et al. 2000, ApJ, 530, 966

Lucy, L. B. 1999, A\&A, 345, 211
Marion, G. H., Höflich, P., Vacca, W. D., et al. 2003, ApJ, 591, 316

Mazzali, P. A., \& Lucy, L. B. 1993, A\&A, 279, 447

Mazzali, P. A., Lucy, L. B., Danziger, I. J., et al. 1993, A\&A, 269, 423

Mazzali, P. A., Danziger, I. J., \& Turatto, M. 1995, A\&A, 297, 509

Mazzali, P. A., Cappellaro, E., Danziger, I. J., Turatto, M., \& Benetti, S. 1998, ApJ, 499, L49

Mazzali, P. A. 2000, A\&A, 363, 705

Nomoto, K., Thielemann, F.-K., \& Yokoi, K. 1984, ApJ, 286, 644

Nugent, P., Phillips, M., Baron, E., et al. 1995, ApJ, 455, L147

Patat, F., Benetti, S., Cappellaro, E., et al. 1996, MNRAS, 278, 111

Phillips, M. M. 1993, ApJ, 413, L105

Phillips, M. M., Lira, P., Suntzeff, N. B., et al. 1999, AJ, 118, 1766

Pignata, G., et al. 2004, MNRAS, accepted

Riess, A. G., Filippenko, A. V., Challis, P., et al. 1998, AJ, 116, 1009

Riess, A., Filippenko, A. V., Li., W., et al. 1999, ApJ, 118, 2675

Röpke, F. K., \& Hillebrandt, W. 2005, A\&A, 429, L29

Ruiz-Lapuente, P., Comeron, F., Mendez, J., et al. 2004 [arXiv: astro-ph/0410673]

Salvo, M. E., Cappellaro, E., Mazzali, P. A., et al. 2001, MNRAS, 321, 254

Shortridge, K. 2002, Star link User Note 86. 20

Smartt, S. J., Patat, F., Meikle, P., \& Araujo, S. 2002, IAUC, 7961

Stehle, M., Mazzali, P. A., Benetti, S., \& Hillebrandt, W. 2004, MNRAS, submitted [arXiv: astro-ph/0409342]

Wheeler, J. C., Höflich, P., Harkness, R. P., \& Spyromilio, J. 1998, ApJ, 496, 908

Wood-Vasey, W. M., Li, W. D., Swift, B., \& Ganeshalingam, M. 2002, IAUC, 7959 